\title{
Analytical expression for the convolution of a Fano line profile with a Gaussian
}

\author{
S. Schippers \\ I. Physikalisches Institut, Justus-Liebig-Universität Gießen, Heinrich-Buff-Ring 16, \\ 35392 Giessen, Germany
}

\begin{abstract}
Asymmetric Fano line profiles are frequently encountered, e.g., in the photoionization spectra of atoms and ions. For the fitting of spectral line profiles to experimental spectra the line profiles have to be convolved with the experimental window function. The latter is often taken to be a Gaussian. It is shown that the convolution can be represented by a rather simple analytic expression involving the Faddeeva function for the evaluation of which efficient and accurate numerical algorithms are available.
\end{abstract}

Keywords: line shapes, Fano profile, convolution, special functions PACS: 32.70.Jz, 02.30.Gp

\section{Introduction}

Asymmetric line profiles are frequently encountered, e.g. in atomic photoionization [1, 2, 3] or photorecombination [4, 5, 6], due to a quantum mechanical interference between resonant and a nonresonant ionization or recombination pathways. According to the quantum mechanical analysis of Fano [7] the photoionization cross section as a function of photon energy $E$ in the vicinity of the resonance energy $E_{\text {res }}$ can be represented as [8]

$$
\sigma(\epsilon)=\sigma_{0}+\sigma_{1} \frac{(q+\epsilon)^{2}}{1+\epsilon^{2}}
$$

Email address: schippers@jlug.de (S. Schippers)

$U R L:$ http://www.uni-giessen.de/amp (S. Schippers) 
where $q$ is the asymmetry parameter and $\sigma_{0}$ and $\sigma_{1}$ are slowly varying functions of the reduced energy $\epsilon=2\left(E-E_{\text {res }}\right) / \Delta_{L}$. Here, $\Delta_{L}$ is the natural (Lorentzian) line width.

For the extraction of the resonance parameters $\sigma_{1}, E_{\mathrm{res}}, \Delta_{L}$ and $q$ from the experimental data, the measured resonance lines can be fitted by a Fano profile. In such fits the experimental photon energy distribution (window function) has to be taken into account. In many cases the experimental window function can be represented as a Gaussian where the Gaussian full width at half maximum (FWHM) corresponds to the experimental energy spread. Thus, for a fit to the experimental data the Fano profile has to be convolved with a Gaussian.

The situation is similar to emission spectroscopy of hot gases where Doppler broadening results in Voigt line profiles, i.e., the convolution of a Lorentzian with a Gaussian. It is well known (see, e.g., [9, 10, 11, 12]) that the Voigt profile can be calculated efficiently from the Faddeeva function [13. 14] (a scaled complex error function). Here, it is shown that also the convolution of a Fano profile with a Gaussian can be represented by the Faddeeva function. The resulting formula allows for a fast and accurate evaluation of the convolution, e.g., in peak fitting routines. It is mentioned that a different, more complex formula has been published earlier without its derivation [15]. It seems, that this formula has not received much attention since even in more recent work the convolution of a Fano profile with a Gaussian has only been treated approximately or by numerical integration [16, 17].

The present paper, which refines an earlier preprint [18], is organized as follows. In section 2 the calculation of the Voigt profile from the Faddeeva function is reviewed. In section 3 some relevant properties of the Faddeeva function are presented. An expression for the convolution of the Fano profile with a Gaussian in terms of the Faddeeva function is derived in section 4 . A conclusive summary is given in section 5 .

\section{The Voigt profile}

The convolution of a Lorentzian line profile

$$
L(E)=A \frac{2}{\pi} \frac{\Delta_{L}}{4\left(E-E_{\text {res }}\right)^{2}+\Delta_{L}^{2}}
$$


with a Gaussian

$$
G(E)=\frac{2}{\Delta_{G}} \sqrt{\frac{\ln 2}{\pi}} \exp \left[-\frac{4(\ln 2) E^{2}}{\Delta_{G}^{2}}\right]
$$

yields the Voigt profile

$$
\begin{aligned}
V(E) & =\int_{-\infty}^{\infty} L\left(E^{\prime}\right) G\left(E^{\prime}-E\right) d E^{\prime} \\
& =A \frac{4 \sqrt{\ln 2}}{\pi^{3 / 2} \Delta_{G}} \int_{-\infty}^{\infty} \frac{\Delta_{L}}{4\left(E^{\prime}-E_{\mathrm{res}}\right)^{2}+\Delta_{L}^{2}} \exp \left[-\frac{4(\ln 2)\left(E-E^{\prime}\right)^{2}}{\Delta_{G}^{2}}\right] d E^{\prime}
\end{aligned}
$$

The profiles in Eqs. 2 and 3 are normalized such that

$$
\int L(E) d E=A \text { and } \int G(E) d E=1 .
$$

The widths $\Delta_{L}$ and $\Delta_{G}$ are the Lorentzian and Gaussian FWHM, respectively. With the definitions

$$
t=\frac{2 \sqrt{\ln 2}\left(E^{\prime}-E\right)}{\Delta_{G}}, x=\frac{2 \sqrt{\ln 2}\left(E_{\mathrm{res}}-E\right)}{\Delta_{G}}, \text { and } y=\frac{\Delta_{L} \sqrt{\ln 2}}{\Delta_{G}},
$$

Eq. 5 transforms into

$$
V(E)=A \frac{2 \sqrt{\ln 2}}{\Delta_{G} \sqrt{\pi}} \frac{1}{\pi} \int_{-\infty}^{\infty} \frac{y e^{-t^{2}}}{(t-x)^{2}+y^{2}} d t=A \frac{2 \sqrt{\ln 2}}{\Delta_{G} \sqrt{\pi}} \Re[w(z)]
$$

where $w(z)$ denotes the Faddeeva function and $z=x+i y$.

\section{Some properties of the Faddeeva function}

The Faddeeva function is a scaled complex error function. It is defined for $\Im z=y>0$ as [13, 14, 19, 20]

$$
w(z)=e^{-z^{2}} \operatorname{erfc}(-i z)=\frac{i}{\pi} \int_{-\infty}^{\infty} \frac{e^{-t^{2}}}{z-t} d t .
$$

Its real and imaginary parts are

$$
\Re[w(z)]=\frac{1}{\pi} \int_{-\infty}^{\infty} \frac{y e^{-t^{2}}}{(t-x)^{2}+y^{2}} d t
$$



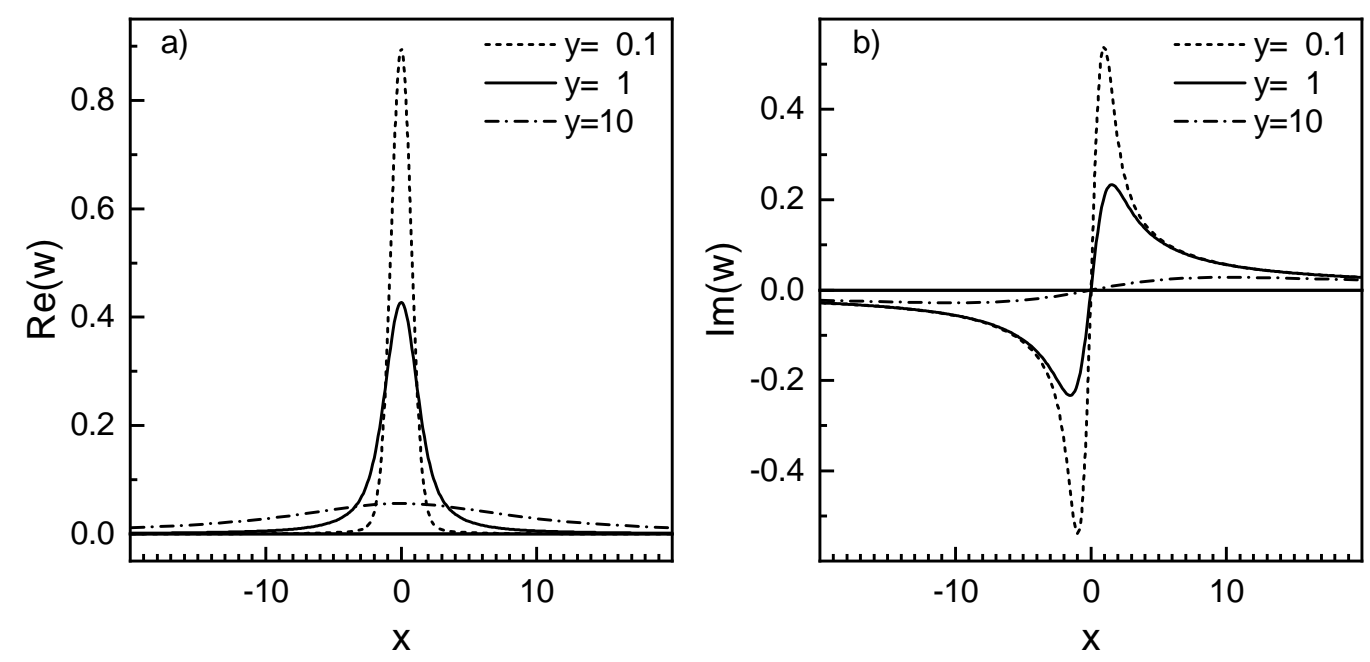

Figure 1: Real part (panel a) and imaginary part (panel b) of the Faddeeva function $w(x+i y)$ as function of the scaled energy $x=2 \sqrt{\ln 2}\left(E_{\mathrm{res}}-E\right) / \Delta_{G}$ for different ratios $y=\Delta_{L} \sqrt{\ln 2} / \Delta_{G}$ of Lorentzian and Gaussian widths. The real part is essentially the Voigt profile (Eq. 7). The imaginary part occurs in addition in the convolution of a Fano profile with a Gaussian (Eq. 26).

and

$$
\Im[w(z)]=\frac{-1}{\pi} \int_{-\infty}^{\infty} \frac{(t-x) e^{-t^{2}}}{(t-x)^{2}+y^{2}} d t .
$$

Figure 1 displays $\Re[w(x+i y)]$ and $\Im[w(x+i y)]$ as functions of the scaled energy $x$ for different ratios $y$ of Lorentzian and Gaussian widths.

For later use we now calculate the integral

$$
I_{2}(x, y)=\frac{1}{\pi} \int_{-\infty}^{\infty} \frac{t^{2} e^{-t^{2}}}{(t-x)^{2}+y^{2}} d t .
$$

To this end we define (cf. Eq. 8)

$$
w_{\eta}(z)=\frac{i}{\pi} \int_{-\infty}^{\infty} \frac{e^{-\eta t^{2}}}{z-t} d t
$$

With this definition

$$
I_{2}(x, y)=\frac{-1}{y} \Re\left[\frac{d w_{\eta}(z)}{d \eta}\right]_{\eta=1}
$$


For the calculation of the derivative on the right-hand side of this equation we exploit the identity $w_{\eta}(z)=w(\sqrt{\eta} z)$ which follows from the substitution $(\sqrt{\eta} t) \rightarrow t$ in Eq. 12 . This yields

$$
\begin{aligned}
\frac{d w_{\eta}(z)}{d \eta} & =\frac{d w(\sqrt{\eta} z)}{d \eta}=\frac{d(\sqrt{\eta} z)}{d \eta} \frac{d w(\sqrt{\eta} z)}{d(\sqrt{\eta} z)} \\
& =\frac{z}{2 \sqrt{\eta}}\left(-2(\sqrt{\eta} z) w(\sqrt{\eta} z)+\frac{2 i}{\sqrt{\pi}}\right)
\end{aligned}
$$

where in the last step we have used the identity [13, 21]

$$
\frac{d w(z)}{d z}=-2 z w(z)+\frac{2 i}{\sqrt{\pi}} .
$$

Combining Eqs. 13 and 14 yields

$$
I_{2}(x, y)=\frac{1}{y} \Re\left[z^{2} w(z)-i \frac{z}{\sqrt{\pi}}\right]=\left(\frac{x^{2}}{y}-y\right) \Re(w)-2 x \Im(w)+\frac{1}{\sqrt{\pi}} .
$$

From equation 15 and by exploiting the Cauchy-Riemann relations, the partial derivatives of $w(z)$ with respect to $x$ and $y$ are easily calculated as

$$
\begin{aligned}
& \frac{\partial \Re(w)}{\partial x}=\frac{\partial \Im(w)}{\partial y}=-2 x \Re(w)+2 y \Im(w), \\
& \frac{\partial \Im(w)}{\partial x}=-\frac{\partial \Re(w)}{\partial y}=-2 y \Re(w)-2 x \Im(w)+\frac{2}{\sqrt{\pi}} .
\end{aligned}
$$

These are useful, e.g., in least-squares fitting routines.

\section{Convolution of a Fano profile with a gaussian}

For the purpose of peak fitting we define the Fano line profile somewhat differently as suggested by Eq. 1, i.e.

$$
F(E)=\frac{2 a}{q^{2} \Delta_{L} \pi}\left[\frac{(q+\epsilon)^{2}}{1+\epsilon^{2}}-1\right]
$$

with a positive amplitude $a>0$. For $q \rightarrow \infty$ the Fano profile as defined by Eq. 19 approaches a symmetric Lorentzian (Eq. 2), i. e. $F(E) \rightarrow L(E)$. 
Moreover, this definition of the Fano profile is consistent with the theoretical treatment of the atomic photoabsorption cross section [22].

With $t, x$ and $y$ from Eq. 6 the convolution with a Gaussian as defined by Eq. 3 can be expressed as

$$
\begin{aligned}
& C(E)= \int_{-\infty}^{\infty} F\left(E^{\prime}\right) G\left(E^{\prime}-E\right) d E^{\prime} \\
&= \frac{2 a}{q^{2} \Delta_{L} \pi}\left[\frac{1}{\sqrt{\pi}} \int_{-\infty}^{\infty} \frac{[q y+(t-x)]^{2} e^{-t^{2}}}{(t-x)^{2}+y^{2}} d t-1\right] \\
&= \frac{2 a}{q^{2} \Delta_{L} \sqrt{\pi}} \times \\
& {\left[\frac{1}{\pi} \int_{-\infty}^{\infty} \frac{\left[q^{2} y^{2}+2 q y(t-x)+t^{2}-2 x(t-x)-x^{2}\right] e^{-t^{2}}}{(t-x)^{2}+y^{2}} d t-\frac{1}{\sqrt{\pi}}\right] } \\
&= \frac{2 a}{q^{2} \Delta_{L} \sqrt{\pi}} \times \\
&\left\{\frac { q ^ { 2 } y \Re ( w ) - 2 q y \Im ( w ) + I _ { 2 } + 2 x \Im ( w ) - \frac { x ^ { 2 } } { y } \Re ( w ) - \frac { 1 } { \sqrt { \pi } } \} } { q ^ { 2 } \Delta _ { L } \sqrt { \pi } } \left\{q^{2} \Re(w)-2 q \Im(w)+\frac{x^{2}}{y^{2}} \Re(w)-\Re(w)-\right.\right. \\
&\left.\quad \frac{2 x}{y} \Im(w)+\frac{1}{y \sqrt{\pi}}+\frac{2 x}{y} \Im(w)-\frac{x^{2}}{y^{2}} \Re(w)-\frac{1}{y \sqrt{\pi}}\right\} \\
&=\frac{a}{q^{2}} \frac{2 \sqrt{\ln 2}}{\Delta_{G} \sqrt{\pi}}\left\{\left(q^{2}-1\right) \Re(w)-2 q \Im(w)\right\} .
\end{aligned}
$$

In this derivation the definitions of $\Re(w)=\Re[w(z)]$ (Eq. 9p, $\Im(w)=\Im[w(z)]$ (Eq. 10), and $I_{2}$ (Eqs. 11 and 16) have been used. It is easily seen that $C(E) \rightarrow V(E)$ for $q \rightarrow \infty$ as expected and that the asymmetry of the profile $C(E)$ is due to the additional term containing the imaginary part $\Im[w(z)]$ (Fig 1 $1 \mathrm{~b}$ ) of the Faddeeva function $w(z)$. Since $\Im[w(z)]$ (Eq. 10) is an odd function of the scaled energy $x$ it does not contribute to the integrated line strength, i.e.

$$
\int_{-\infty}^{\infty} C(E) d E=\int_{-\infty}^{\infty} V(E) d E
$$

holds. From the comparison of the real part of $C(E)$ (Eq. 22) with the Voigt profile $V(E)$ (Eq. 7) one obtains the following relation between the peak area 
$A$ and the amplitude $a>0$ :

$$
A=a \frac{q^{2}-1}{q^{2}}
$$

For $|q|<1$ the peak area becomes negative. This indicates that the resonance profile produces a dip (window resonance) in the absorption cross section [22]. Inserting Eq. 24 in Eqs. 19 and 22 finally yields

$$
F(E)=\left|\frac{A}{q^{2}-1}\right| \frac{2}{\Delta_{L} \pi}\left[\frac{(q+\epsilon)^{2}}{1+\epsilon^{2}}-1\right]
$$

and

$$
C(E)=\left|\frac{A}{q^{2}-1}\right| \frac{2 \sqrt{\ln 2}}{\Delta_{G} \sqrt{\pi}}\left\{\left(q^{2}-1\right) \Re[w(z)]-2 q \Im[w(z)]\right\} .
$$

\section{Summary}

It has been demonstrated that the convolution of a Fano line profile (Eq. 25) with a Gaussian (Eq. 3) can be evaluated analytically (Eq. 26) by using the Faddeeva function (scaled complex error function) $w(z)$ with $z=x+i y$ and with $x$ and $y$ from Eq. 6. Various fast and accurate algorithms for computing the Faddeeva function have been described in the literature [9, 10, 12, 23, 24, 25, 26, 27, 28]. Their performances have been critically evaluated repeatedly [11, 12, 29, 30. According to the findings of Zaghloul and Ali [12] their algorithm is the most accurate to date. An implementation of this algorithm in the Fortran programming language has been published [31] and a modified version of this algorithm coded in the programming language $\mathrm{C}^{++}$is available from the internet [32].

The line profile $C(E)$ (Eq. 26) has been implemented by the author as a user-supplied fit function for the commercial software Origin [33]. The implementation is available from the author upon request. It has already been

successfully used in various contexts [e.g. [5, 34, 35]. It should be noted that the here introduced derivations can also be applied to related line shapes. An example has been provided recently in the context of precision spectroscopy of atomic hydrogen [36].

\section{Acknowledgments}

The author would like to thank Alfred Müller and Thomas Udem for helpful discussions. 


\section{References}

[1] K. Schulz, G. Kaindl, M. Domke, J. D. Bozek, P. A. Heimann, A. S. Schlachter, J. M. Rost, Observation of new Rydberg series and resonances in doubly excited helium at ultrahigh resolution, Phys. Rev. Lett. 77 (1996) 3086-3089. doi:10.1103/PhysRevLett.77.3086.

URL https://doi .org/10.1103/PhysRevLett.77.3086

[2] H. Kjeldsen, Photoionization cross sections of atomic ions from mergedbeam experiments, J. Phys. B 39 (2006) R325-R377. doi:10.1088/ 0953-4075/39/21/R01.

URL https ://doi .org/10.1088/0953-4075/39/21/R01

[3] S. Schippers, A. L. D. Kilcoyne, R. A. Phaneuf, A. Müller, Photoionization of ions with synchrotron radiation: From ions in space to atoms in cages, Contemp. Phys. 57 (2016) 215-229. doi : 10.1080/00107514. 2015.1109771.

URL https : //doi .org/10.1080/00107514.2015.1109771

[4] D. A. Knapp, P. Beiersdorfer, M. H. Chen, J. H. Scofield, D. Schneider, Observation of interference between dielectronic recombination and radiative recombination in highly charged uranium ions, Phys. Rev. Lett. 74 (1995) 54-57. doi:10.1103/PhysRevLett.74.54.

URL https://doi.org/10.1103/PhysRevLett.74.54

[5] S. Schippers, S. Kieslich, A. Müller, G. Gwinner, M. Schnell, A. Wolf, M. Bannister, A. Covington, L. B. Zhao, Interference effects in the photorecombination of argonlike $\mathrm{Sc}^{3+}$ ions: Storage ring experiment and theory, Phys. Rev. A 65 (2002) 042723. doi:10.1103/PhysRevA.65. 042723 .

URL https://doi.org/10.1103/PhysRevA.65.042723

[6] A. J. González Martínez, J. R. Crespo López-Urrutia, J. Braun, G. Brenner, H. Bruhns, A. Lapierre, V. Mironov, R. Soria Orts, H. Tawara, M. Trinczek, J. Ullrich, J. H. Scofield, State-selective quantum interference observed in the recombination of highly charged $\mathrm{Hg}^{75+-78+}$ mercury ions in an electron beam ion trap, Phys. Rev. Lett. 94 (2005) 203201. doi:10.1103/PhysRevLett.94.203201. URL https://doi.org/10.1103/PhysRevLett.94.203201 
[7] U. Fano, Effects of configuration interaction on intensities and phase shifts, Phys. Rev. 124 (1961) 1866-1878. doi:10.1103/PhysRev.124. 1866.

URL https ://doi .org/10.1103/PhysRev.124.1866

[8] U. Fano, J. W. Cooper, Line profiles in the far-UV absorption spectra of the rare gases, Phys. Rev. 137 (1965) A1364-A1379. doi:10.1103/ PhysRev.137.A1364.

URL https ://doi .org/10.1103/PhysRev.137.A1364

[9] J. Humlícek, An efficient method for evaluation of the complex probability function: The Voigt function and its derivatives, J. Quant. Spectrosc. Radiat. Transfer 21 (1979) 309-313. doi:10.1016/0022-4073(79) 90062-1.

URL https://doi .org/10.1016/0022-4073(79)90062-1

[10] R. J. Wells, Rapid approximation to the Voigt/Faddeeva function and its derivatives, J. Quant. Spectrosc. Radiat. Transfer 62 (1999) 29-48. doi:10.1016/S0022-4073(97)00231-8.

URL https://doi.org/10.1016/S0022-4073(97)00231-8

[11] F. Schreier, Optimized implementations of rational approximations for the Voigt and complex error function, J. Quant. Spectrosc. Radiat. Transfer 112 (2011) 1010 - 1025. doi:10.1016/j.jqsrt.2010.12.010. URL https ://doi .org/10.1016/j.jqsrt.2010.12.010

[12] M. R. Zaghloul, A. N. Ali, Algorithm 916: Computing the Faddeyeva and Voigt functions, ACM Trans. Math. Softw. 38 (2011) 15. doi: 10.1145/2049673.2049679.

URL https://doi .org/10.1145/2049673.2049679

[13] M. Abramowitz, I. A. Stegun, Handbook of Mathematical Functions, Dover Publications, New York, 1964, chapter 7.

[14] Digital Library of Mathematical Functions, National Institute of Standards and Technology, Gaithersburg, Maryland, USA, Release date 2011-08-29, http://dlmf.nist.gov/7.7.E2.

URL http://dlmf .nist.gov/7.7.E2

[15] T. K. Fang, T. N. Chang, Determination of profile parameters of a Fano resonance without an ultrahigh-energy resolution, Phys. Rev. A 
57 (1998) 4407-4412. doi:10.1103/PhysRevA.57.4407.

URL https://doi.org//10.1103/PhysRevA.57.4407

[16] C. M. Teodorescu, J. M. Esteva, R. C. Karnatak, A. E. Afif, M. Womes, Quantitative analysis of the sodium 1s single- and double-excitation spectrum by using atomic profiles convolved with the instrumental function, J. Phys. B 30 (1997) 4293. doi:10.1088/0953-4075/30/19/018. URL https : //doi .org/10.1088/0953-4075/30/19/018

[17] X.-J. Liu, Y.-P. Huang, L.-F. Zhu, Z.-S. Yuan, W.-B. Li, K.-Z. Xu, Numerical determination of profile parameters for Fano resonance with definite energy resolution, Nucl. Instrum. Methods A 508 (2003) 448 453. doi:10.1016/S0168-9002(03)01659-0. URL https : //doi.org/10.1016/S0168-9002(03)01659-0

[18] S. Schippers, Analytical expression for the convolution of a Fano line profile with a Gaussian, ArXiv e-prints 1203.4281v2, also available as S. Schippers, Int. Rev. At. Mol. Phys. 2, 151 (2011) from http://www.auburn.edu/cosam/departments/physics/iramp/2_2/index.htm. URL https://arxiv.org/abs/1203.4281

[19] G. P. M. Poppe, C. M. J. Wijers, More efficient computation of the complex error function, ACM Trans. Math. Softw. 16 (1990) 38-46. doi : 10.1145/77626.77629. URL https://doi.org/10.1145/77626.77629

[20] M. Sampoorna, K. Nagendra, H. Frisch, Generalized Voigt functions and their derivatives, J. Quant. Spectrosc. Radiat. Transfer 104 (2007) 71 85. doi:10.1016/j.jqsrt.2006.08.011. URL https://doi.org/10.1016/j.jqsrt.2006.08.011

[21] Digital Library of Mathematical Functions, National Institute of Standards and Technology, Gaithersburg, Maryland, USA, Release date 2011-08-29, http://dlmf.nist.gov/7.10.E2.

URL http://dlmf .nist.gov/7.10.E2

[22] J. M. Rost, K. Schulz, M. Domke, G. Kaindl, Resonance parameters of photo doubly excited helium, J. Phys. B 30 (1997) 4663. doi:10.1088/ 0953-4075/30/21/010.

URL https://doi.org/10.1088/0953-4075/30/21/010 
[23] W. Gautschi, Algorithm 363: Complex error function, Comm. ACM 12 (1969) 635. doi:10.1145/363269.363618.

URL https : //doi .org/10.1145/363269.363618

[24] W. Gautschi, Efficient computation of the complex error function, SIAM J. Num. Anal. 7 (1970) 187-198. doi:10.1137/0707012.

URL https ://doi .org/10.1137/0707012

[25] J. Humlícek, Optimized computation of the Voigt and complex probability functions, J. Quant. Spectrosc. Radiat. Transfer 27 (1982) 437-444. doi:10.1016/0022-4073(82)90078-4.

URL https://doi .org/10.1016/0022-4073(82)90078-4

[26] G. P. M. Poppe, C. M. J. Wijers, Algrorithm 680: Evaluation of the complex error function, ACM Trans. Math. Softw. 16 (1990) 47. doi: 10.1145/77626.77630.

URL https ://doi .org/10.1145/77626.77630

[27] K. L. Letchworth, D. C. Benne, Rapid and accurate calculation of the Voigt function, J. Quant. Spectrosc. Radiat. Transfer 107 (2007) 173192. doi:10.1016/j.jqsrt.2007.01.052.

URL https://doi.org/10.1016/j.jqsrt.2007.01.052

[28] S. Abrarov, B. Quinea, R. Jagpal, A simple interpolating algorithm for the rapid and accurate calculation of the Voigt function, J. Quant. Spectrosc. Radiat. Transfer 110 (2009) 376-383. doi:10.1016/j.jqsrt. 2009.01.003.

URL https://doi.org/10.1016/j.jqsrt.2009.01.003

[29] F. Schreier, The Voigt and complex error function: A comparison of computational methods, J. Quant. Spectrosc. Radiat. Transfer 48 (1992) 743-762. doi:10.1016/0022-4073(92)90139-U. URL https://doi .org/10.1016/0022-4073(92)90139-U

[30] F. Schreier, D. Kohlert, Optimized implementations of rational approximations-a case study on the Voigt and complex error function, Comput. Phys. Commun. 179 (2008) 457-465. doi:10.1016/j.cpc. 2008.04 .012 .

URL https://doi.org/10.1016/j.cpc.2008.04.012 
[31] M. R. Zaghloul, Remark on "Algorithm 916: Computing the Faddeyeva and Voigt functions": Efficiency improvements and Fortran translation, ACM Trans. Math. Softw. 42 (2016) 26:1-26:9. doi:10.1145/2806884. URL https : //doi .org/10.1145/2806884

[32] S. G. Johnson, Faddeeva Package, Massachusetts Institute of Technology, Cambridge, Massachusetts, USA (2012).

URL http://ab-initio.mit.edu/wiki/index.php/Faddeeva_ Package

[33] Origin, OriginLab Corporation, One Roundhouse Plaza, Suite 303 , Northampton, MA 01060, USA, version 7.5 and higher.

URL https: //www .originlab.com/

[34] S. Schippers, A. Müller, B. M. McLaughlin, A. Aguilar, C. Cisneros, E. Emmons, M. F. Gharaibeh, R. A. Phaneuf, Photoionization studies of the $\mathrm{B}^{+}$valence shell: Experiment and theory, J. Phys. B 36 (2003) 3371-3381. doi:10.1088/0953-4075/36/16/301. URL https : //doi .org/10.1088/0953-4075/36/16/301

[35] S. W. J. Scully, I. Álvarez, C. Cisneros, E. D. Emmons, M. F. Gharaibeh, D. Leitner, M. S. Lubell, A. Müller, R. A. Phaneuf, R. Püttner, A. S. Schlachter, S. Schippers, B. M. McLaughlin, Doubly excited resonances in the photoionization spectrum of $\mathrm{Li}^{+}$: Experiment and theory, J. Phys. B 39 (2006) 3957-3968. doi:10.1088/0953-4075/39/18/024.

URL https : //doi .org/10.1088/0953-4075/39/18/024

[36] A. Beyer, L. Maisenbacher, A. Matveev, R. Pohl, K. Khabarova, A. Grinin, T. Lamour, D. C. Yost, T. W. Hänsch, N. Kolachevsky, T. Udem, The Rydberg constant and proton size from atomic hydrogen, Science 358 (2017) 79-85. doi:10.1126/science.aah6677.

URL https://doi.org/10.1126/science.aah6677 